\title{
Analysis of a Rectangular Microstrip Patch Antenna with EBG Structures
}

\author{
Gaurav Kumar Sharma \\ M.Tech. Scholar \\ Om Parkash Floor Mill Ajit Nagar Verka, \\ AMRITSAR, 143501
}

\author{
Narinder Sharma \\ Associate Professor \\ Amritsar College Of Engg. \& Tech \\ AMRITSAR
}

\begin{abstract}
In this paper, the execution of rectangular patch antenna is mended applying a newly cylindrical electromagnetic bandgap (EBG) substrate. The microstrip patch antenna is fed by the driven terminal. This EBG structure, when comprised with microstrip patch antenna to radiation pattern and return loss got from menstruation appearance a better impedance matching and a gain enhancement of the aimed antenna. The gain using the designed structure has been found to be $7.73 \mathrm{~dB}$, which are merits for transmitting data over a long distance. Wideband antenna finds its applications in mobile sensors, data ingathering and tracking applications.
\end{abstract}

\section{Keywords}

Microstrip patch antenna, electromagnetic band gap structure, gain.

\section{INTRODUCTION}

Microstrip patch antennas are the almost mutual form of printed antennas. A microstrip patch antenna is widely utilized in covenant and portable communication devices ascribable to its little size, dilute profile configurations, accordance and low price. Hence it can be employed in a broad form of applications roaming from mobile communication to satellite, aircraft and other applications [1]. The patch antennas gain can be enhanced by applying multiple patches attached to an array or by coming down the surface wave which can produce ripples in the radiation pattern. Various methods have been aimed to bring down the consequences of surface waves. One approach path proposed is the synthesized substrate that lets down the effectual dielectric constant of the substrate either below or all over the patch [2-3]. Other approach paths are to apply parasitic elements [4-5] or to apply a minimized surface-wave antenna [6]. Electromagnetic band-gap (EBG) structures, also known as photonic crystals [7], are as well applied to better the antenna functioning [8-14]. These structures also have the power to open a band gap, which is a frequency range for which the generation of electromagnetic waves is prevented. By smothering a patch antenna with a square-lattice of small metal pads with grounding vias, also called mushroom-like structure, a significant suppression of surface waves excited in the dielectric substrate has been detected, which better the gain of the antenna or efficient radiated ability [9]. Reduce of mutual coupling and co-site interference is other merits of this EBG antenna. The inbuilt drawbacks admit constrict bandwidth and relative prominent size. The requirement of a more minor antenna size much struggle with the desired wide band or multiband applications. Reconfigurable or tunable antennas supply potential results and have appealed significantly research attempts [1-4]. Instead than backing wide-band or multiband performances at the same time, accredited antennas are controlled to hop over unlike bands dynamically. Such an antenna would not back all bands simultaneously, but it allows constrict instant bandwidth that is selectable dynamically.

Since antenna radiation features, incubate resonant frequency and radiation patterns are ascertained for the most part by its shapes and size, altering its geometry mechanically allow for a non-rational method to reconfigure its radiation attributes. MEMS techniques incorporate electrical and mechanical operates in a single component using micro fabrication or micro aching technology, and can be recognized with present semiconductor integrated circuit serving [5-7]. Hence, MEMS based reconfigurable antennas had been widely inquired [813]. By using printed circuit board (PCB) techniques, planar wire antennas in the form of filamentary conductive traces can be constructed with inserted MEMS switches. A MEMS switch converts dynamically the antenna length and enables dual or multiband applications.

Antenna reconfiguration can also be projected electronics switch such as PIN diodes, which are ordinarily used in modern communications and radar applications. When the diode switch is DC forward biased, it is at an "on" state and is ideally a short circuit; while when the diode is reverse biased, it is at an "off" state and the diode is open-circuited. Placing a PIN diode in between two conducting traces, could control electronically the efficient antenna physical length. Planar microstrip antennas using PIN diode switching for reconfiguration had also been investigated [4-8].

Most reconfigurable antennas are grounded on the dynamic control of the antenna physical length. It is possible to design electronically tunable antennas by adapting the attributes of the material where an antenna occupies. Ferrite-

Loaded microstrip antennas are examples [2], where the antenna features are aligned by the external magnetic fields issued by a dc biased circuit.

Electromagnetic band gap (EBG) structures in printed circuits are thin complex dielectric layers with periodic metallic patterns (generally backed by a metal ground plane), and have one or multiple frequency band-gaps in which no substrate mode can exist. This unique ownership has been utilized to design antenna systems with a improve gain and efficiency, lower side-lobes and back-lobe levels amend isolations within array elements, by crushing surface wave modes [2-4]. The EBG substrate has been utilized to alleviate some withdraws of conventional microstrip antennas [5-7]. Recently, radiation features of a microstrip patch over a mushroom-type electromagnetic band-gap (EBG) substrate were inquired [9]. The EBG surface $e$ is found to have the impressions of diluting the patch resonant length and bandwidth as a result of stronger capacitive coupling.

In this paper, the functioning of a rectangular microstrip patch antenna has been mended utilizing a new cylindrical electromagnetic band-gap (EBG) substrate. The 
patch antenna is fed by a driven terminal and is integrated within a cylindrical electromagnetic band-gap substrate, based on the mushroom-like substrate, to raise the antenna gain. The cylindrical electromagnetic band-gap structure applied is a combination of two periodic structures with dissimilar periods. The feature of the patch antenna based on drilling air holes and embedding a metal boundary in the substrate were studied by [9]. The outcome suggested that the surface waves which spread along the surface of the substrate can be inhibited by the multiple photonic band-gap structure because of its consequences of forbidden band, that it can diversify almost of electromagnetic waves' energy in the substrate importantly, and that it has lower return loss (S11) compared to the conventional patch antennas and enhanced gain.

\section{ELECTROMAGNETIC BAND-GAP STRUCTURE}

Periodic structures are ample in cosmos beguiled artists and scientists alike. When an electromagnetic waves energizing procedures amazing boasts result.

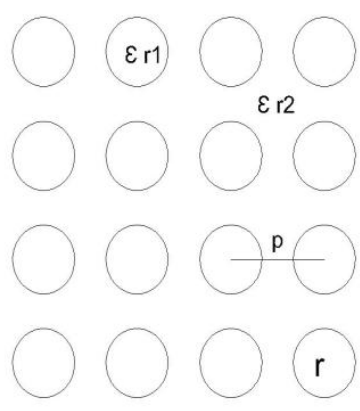

$$
\begin{aligned}
& p: \text { periodicity } \\
& r: \text { radius of unit cell } \\
& \mathcal{E}_{n} \text { permittivity of unit cell } \\
& \mathcal{E}_{2} \text {. permittivity of substrate }
\end{aligned}
$$

Fig 1: Electromagnetic band-gap fundamental structure showing radius and periodicity of structure

In detail, boats frequency stop-bands, pass-bands, and band identified [1]. Brushing up the literature, on several nomenclatures have been applied looking of the applications. These applications are innovation, gratings, frequency exclusive surface photonic crystals [2] and photonic bandgaps They can be assorted beneath the liberal Electromagnetic Band-gap (EBG) structures in General speaking, electromagnetic banish are specified as unreal periodic (or formerly objects that forbid/aid the propagation of waves in a assigned band of frequency for al and all polarization states (as shown in $\mathrm{F}$ structures are commonly recognized by periodic dielectric materials and metallic conductors (as shown in fig. 1). In universal, they can be categorized in allowing to their geometric configuration: (1) dimensional volumetric structures, (2) two-dimensional surfaces, and (3) one-dimensional.

\section{ANALYSIS AND DESIGN}

In this division, a rectangular microstrip patch antenna and its cylindrical EBG substrate are projected by applying a Finite Element method (HFSS-Ansoft). In the pretense operation convergence and minimization of numerical errors were received by assuring that the mesh was sufficiently alright. In Subsection A, the patch antenna is planned to function at an arbitrary chosen frequency. Then, in Subsection B, the EBG substrate is projected to enhance the gain of the patch antenna at its functioning frequency.

\subsection{Rectangular microstrip patch antenna}

This subsection identifies the patch-antenna configuration that is chose to execute the comparison between using a normal substrate and a cylindrical EBG substrate. The antenna is depicted in Fig. 2. The patch antenna is rectangular with length of $1=15.8 \mathrm{~mm}$ and breath is $\mathrm{b}=8 \mathrm{~mm}$ printed on a substrate with a relative permittivity of or $=2.2$. The patch is fed by a probe and placed $6 \mathrm{~mm}$ from the center of the patch. The feed location was optimized to afford better impedance matching. The sizing of the substrate is $180 \mathrm{~mm} \times 180 \mathrm{~mm}$. The antenna resonates at $10.667 \mathrm{GHz}$.

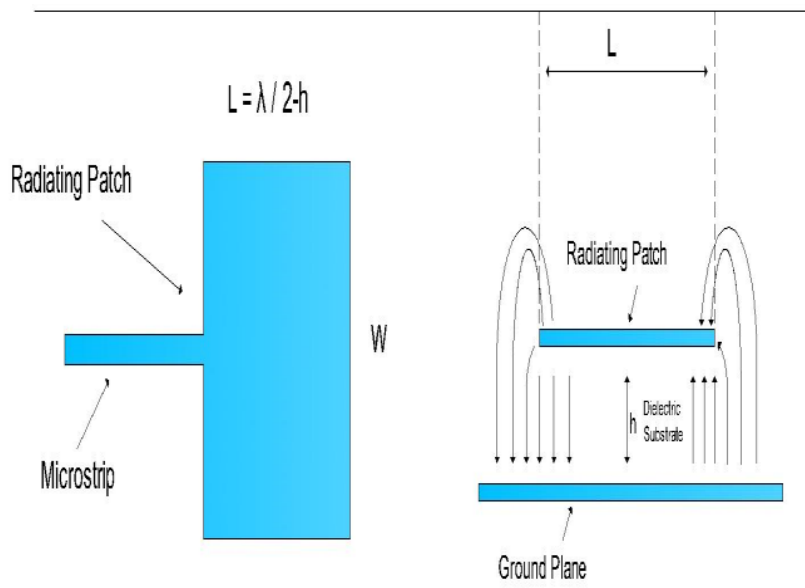

Fig 2: Rectangular patch antenna with microstrip.

The maximum gain of the patch antenna is about $6 \mathrm{~dB}$. The gain of this antenna is heightening using a cylindrical EBG substrate, which is depicted in the next subsection.

\subsection{Patch antenna surrounded by a cylindrical EBG substrate}

While the previous subsection has defined the patch-antenna configuration, the design of the EBG substrate is now presented. Figure 3 shows the schematic of the proposed patch antenna surrounded by a cylindrical structure composed of metal rings and grounding vias. The concentric rings of strips are etched on the same plane than the patch antenna, with the distance $5 \mathrm{~mm}$ from one to another. The first metal ring starts at the radius $2 \mathrm{~mm}$.

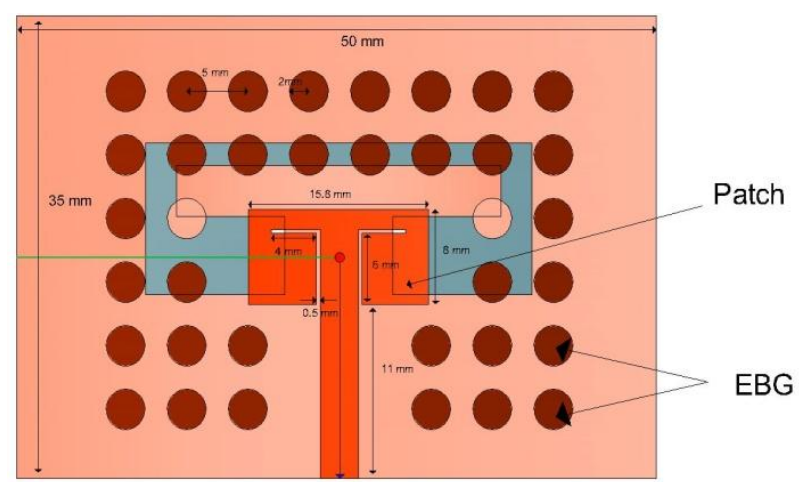

Fig 3: Patch incorporated with the cylindrical EBG substrate. 
The vias have the radius a, and they are disposed with the same transversal period $\mathrm{Pt}$ and the same radial period $\operatorname{Pr} 2$.The following parameters are fixed: $2 \mathrm{a}=\mathrm{g}=2 \mathrm{~mm}$. The remaining parameters Pr1 and Pr2 were optimized. Three concentric rings and three circularly periodic structures of vias are considered. According to numerical results (not shown here), the gain is not sufficiently increased when only one or two layers are used.

\section{SIMULATION RESULT}

The proposed EBG-based antenna structure has been simulated using cell size $129 \times 131 \times 34$. Here, time step $\Delta \mathrm{t}=$ $2.0747 \mathrm{~ns}$. Results have been analyzed for variation of gain and reflection loss because EBG structures are expected to increase gain by suppression of surface waves. fig. 4 show band-gap of the designed EBG structure. The radiation patterns of the proposed design in E-plane and $\mathrm{H}$ - plane respectively are shown in Fig. 5-6.

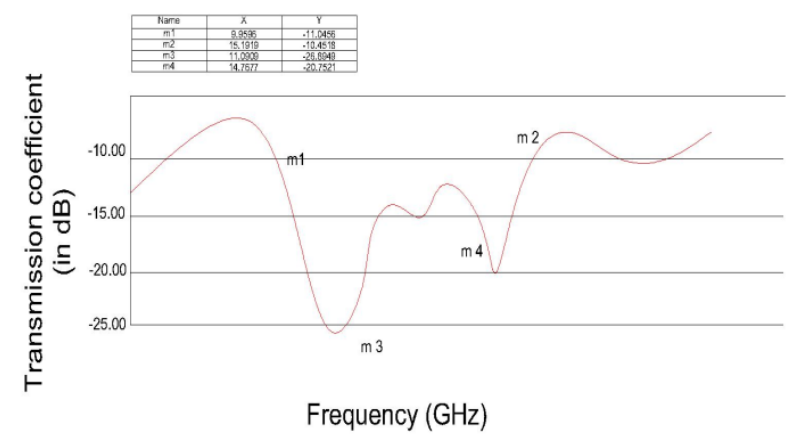

Fig 4: Transmission coefficients (in dB) vs. frequency (in $\mathrm{GHz}$ ) for the proposed structure.
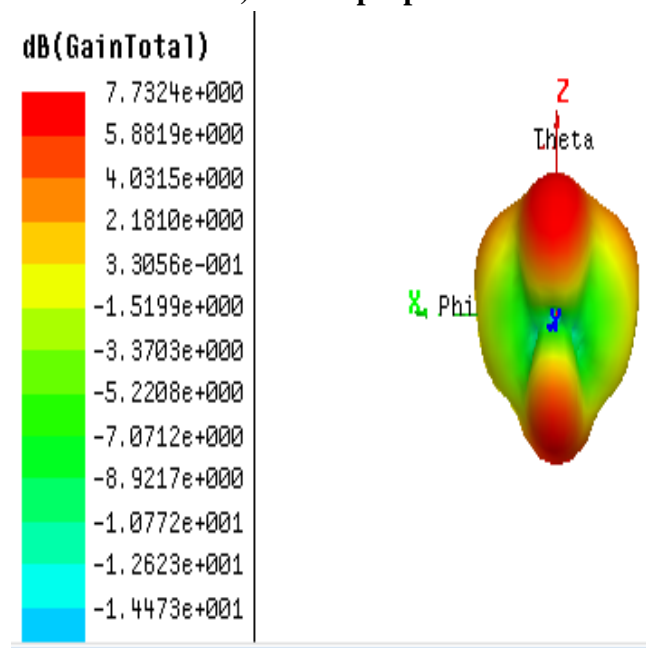

Fig 5: Radiation pattern of antenna with EBG structure at angle 90.
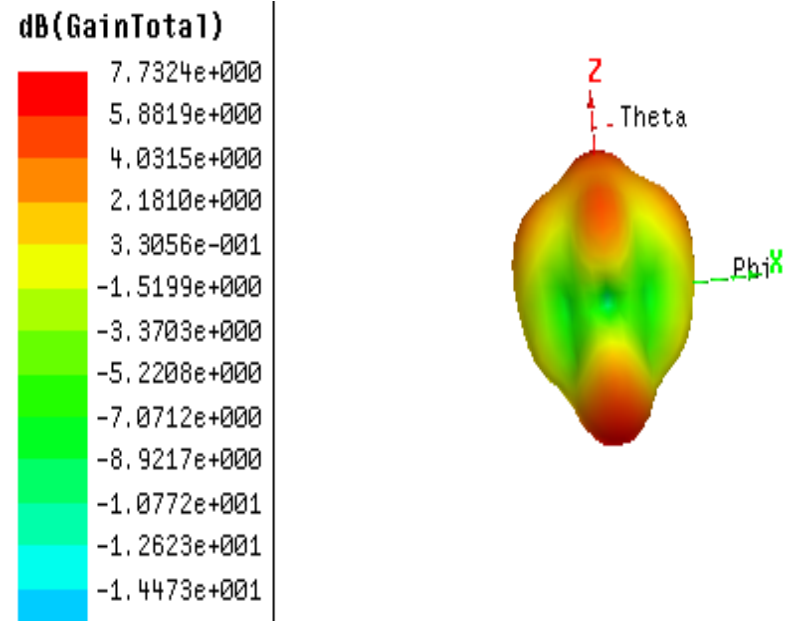

Fig 6: Radiation pattern of antenna with EBG structure at angle 180 .

\section{OBSERVATIONS}

Observations concerning the gain and reflection loss using the proposed antenna structure have been talked about and amended in this section.

\subsection{Gain}

Gain has been accounted from the radiation patterns in $\mathrm{E}$ and $\mathrm{H}$-plane for the patch antenna. Gain receive from proposed antenna structure (EBG substrate) is found to be $7.7324 \mathrm{~dB}$ at $10.667 \mathrm{GHz}$ (from Fig. 5-6). Gain receive from patch without any EBG structures show a value of $6 \mathrm{~dB}$ at an operating frequency of $10.667 \mathrm{GHz}$. Gain obtained using proposed structure is importantly more than that without EBG.

\subsection{Reflection loss}

Reflection loss for aimed EBG-substrate patch is found to attain a lowest value of $-33 \mathrm{~dB}$. It is evident from the reflection loss curves of the antenna structures using EBG and that without EBG that reflection loss has reduced drastically for patch with EBG. This has ensued in a noteworthy improvement in gain. The bandwidth of the constitute EBGbased patch is bumped to be $112.3 \mathrm{MHz}$.

\section{DISCUSSION}

It is known that the gain of the antenna can be enhanced by contracting any loss of the antenna. Selection of an effective quality (low-loss) substrate will contract the dielectric and conductor losses. However, surface waves go inside the substrate and reduce the gain. To reduce this surface wave loss, wave propagation has to be stopped inside or reflected outside the substrate to enhance the gain in forward direction. The waves are arrested or retarded by obstructing their path with a high propagation-impedance - air $(\varepsilon \mathrm{r}=1)$. The impedance offered by a dielectric is given as $\eta / 2 \sqrt{\epsilon_{r}}$. This implies that the impedance offered, is maximum for smallest value of $\varepsilon r$ (the smallest value of $\mathrm{gr}$ being 1 for air). In the proposed antenna, the surface waves are reduced by drilling holes in periodic fashion in the dielectric substrate. The remarkable improvement in gain can be attributed to the periodic effect of embedding air voids (holes) in the substrate.

\section{CONCLUSION}

In this paper, a novel proficiency for the gain sweetening of micro-strip patch antennas using a cylindrical EBG structure has been aimed and established. The EBG structure is based 
on the mushroom-like structure with a circular proportion. It is framed of a periodic structure of metallic rings and of a periodic structure of vias. The periods of time of these structures have been optimized applying a full-wave method to maximize the antenna gain. The new substrate comes down the surface wave, but the gain enhancement is primarily due to the coupling between the patch and the EBG structure. To validate the aimed concept, experimental effects have been demonstrated, showing that a gain enhancement of $2.9 \mathrm{~dB}$ is attained with the new substrate.

\section{REFERENCES}

[1] E. Yablonvitch, "Photonic band-gap structures," J. Opt. Soc. Amer. B, Opt. Physics, vol. 10, no. 2, pp. 283-295, Feb 1993.

[2] G. P. Gauthier, A. Courtay, and G. H. Rebeiz "Microstrip antennas.

[3] M. Khayat, J. T. Williams, D. R. Jakson, and S. A. Long, "Mutual coupling between reduced surface-wave microstrip antennas, IEEE Trans. Antennas Propag., vol.48, pp. 1581-1593, Oct. 2000.

[4] D.M. Kokotoff, R.B. Waterhouse, C.R. Britcher, and J.T. Aberle, "Anular ring coupled circular patch with enhanced performance", Electron. Letters, vol.33, pp. 2000-2001, Nov. 1997.

[5] R.G. Rojas, and K.W. Lee, "Surface wave control using non periodic parasitic strips in printed antennas", IEE Pros.-Microw. Antennas Propag., vol.148, pp. 25-28, Feb. 2001

[6] A. K. Bhattacharayya, "Characteristics of space and surface-waves in a multilayered structure", IEEE Trans. Antennas Propag., vol.38, pp. 1231- 1238, Aug. 1990.
[7] J. Joannopoulos, R. D. Meade and J. N. Winn, Photonic crystals: molding the flow of light, Princeton University Press, 1995M.

[8] R. Gonzalo, P. de Maagt, and M. Sorolla, "Enhanced path-antenna performance by supresing surface waves using photonic-bandgap substrates", IEEE Tran. Microw. Theo. Techn., vol. 47, pp. 2131-2138, Nov. 1999.

[9] D. Sievenpiper, L. Zhang, R.F. Jimenez Broas, N. G. Alexopoulos, and E. Yablonovitch, "High-impedance electromagnetic surfaces with a forbidden frequency band", IEEE Trans. Microwave Theory Techn., vol. 47, pp. 2059-2074, Nov. 1999.

[10] Y.-J. Park, A. Herchlein, and W. Wiesbeck, "A Photonic Bandgap (PBG) structure for guiding and suppressing surface waves in millimeter-wave antennas", IEEE Trans. Antennas Propagat., vol. 49, pp. 1854-1857, Oct. 2001.

[11] K. Agi, M. Mojahedi, B. Minhas, E. Schamilogu, and K. J. Malloy, "The effects of an electromagnetic crystal substrate on a microstrip patch antenna", IEEE Trans. Antennas Propag., vol. 50, pp. 451-456, April 2002.

[12] F. Yang and Y. Rahmat-Samii, "Microstrip antennas integrated with electromagnetic bandgap (EBG) structures: a low mutual coupling design for array applications", IEEE Trans. Antennas Propag., vol. 51, pp. 2936-2946, oct. 2003.

[13] N. Llombart, A. Neto, G. Gerini, and P. de Maagt, "Planar circularly symettric EBG structures for reducing surface waves in printed antennas", IEEE Trans. Antennas Propag., vol. 53, pp. 3210-3218, Oct. 2005.

[14] A. Neto, N. Llombart, G. Gerini, and P. de Maagt, "On the optimal radiation bandwidth of printed slot antennas surrounded by EBGs", IEEE Trans. Antennas Propag., vol. 543, pp. 1074-1083, Ap. 2006. 